\title{
B-Spline Curve Fitting Using Dominant Points*
}

\author{
Hyungjun Park ${ }^{1}$ and Joo-Haeng Lee ${ }^{2}$ \\ ${ }^{1}$ Chosun University, Gwangju 501-759, Korea \\ hzparkachosun.ac.kr \\ ${ }^{2}$ Electronics and Telecommunications Research Institute, Daejeon 305-350, Korea \\ joohaeng@etri.re.kr
}

\begin{abstract}
Motivated by an insight that properly selected points, called dominant points, can play an important role in producing better curve approximation, we propose a new approach for B-spline curve fitting to a sequence of points. The approach is substantially different from the conventional ones in knot placement and dominant point selection. It can generate a B-spline curve in good quality with less deviation. Adopted in the error-bounded curve approximation, it can play an important role in generating B-spline curves with much less control points.
\end{abstract}

\section{Previous Work}

Least-squares B-spline curve fitting has been used as a basic tool to construct a Bspline curve $\mathbf{c}(t)$ from points $\mathbf{p}_{i}(i=0, . ., m)$, which takes three steps: parameterization, knot placement, and the least-squares minimization [1]. In parameterization, we select the parameters $\overline{t_{i}}$ of the points $\mathbf{p}_{i}$. The chord length or centripetal methods have been widely adopted for this task. In knot placement, we determine a knot vector $\mathbf{T}=\left\{t_{0}, t_{1}, \ldots, t_{n+p-1}, t_{n+p}\right\}$ after specifying the order $($ degree +1$) p$ and the index $n$. In the least-squares minimization, we determine control points $\mathbf{b}_{j}(j=0, . ., n)$ of a B-spline curve $\mathbf{c}(t)$ by minimizing the least-squares error $E\left(\mathbf{b}_{0}, \ldots, \mathbf{b}_{n}\right)=\sum_{i=0}^{m}\left\|\mathbf{c}\left(\bar{t}_{i}\right)-\mathbf{p}_{i}\right\|^{2}$. This minimization problem is transformed into the problem of solving a linear system. The knots are determined to reflect the distribution of the parameters. The interior knots $t_{i}$ of the knot vector $\mathbf{T}$ can be spaced as follows [1]:

$$
t_{p+i-1}=\left\{\begin{array}{l}
\frac{1}{p-1} \sum_{j=i}^{i+p-2} \bar{t}_{j} \text { for } m=n \\
(1-u) \bar{t}_{k-1}+u \bar{t}_{k} \text { for } m>n
\end{array} \quad(i=1, . ., n-p+1)\right.
$$

where $k=\operatorname{int}(i \times d), d=(m+1) /(n-p+2)$, and $u=i \times d-k$. This is called the averaging technique (AVG) for $m=n$, and the knot placement technique (KTP) for $m>n$. These techniques give a stable system of equations [1]. Nonetheless, not all stable equations yield acceptable curves. When $m$ is nearly greater than $n(|m-n|$ is small),

\footnotetext{
* This work was supported in part by research funds from Chosun University, 2004.
} 
it is liable to generate undesirable results. To avoid this, Piegl and Tiller [2] suggested another knot placement technique (NKTP).

\section{Proposed Approach}

The proposed approach takes four main steps: parameterization, dominant point selection, knot placement, and least-squares minimization. The approach is substantially different from the conventional ones in knot placement and dominant point selection.

\subsection{Determination of Knots}

Consider that dominant points $\mathbf{d}_{j}(j=0, . ., n)$ have been selected among the given points $\mathbf{p}_{k}(k=0, . ., m)$. Generally, $n$ is not greater than $m(n \leq m)$. With the points $\mathbf{d}_{j}$, interior knots $t_{i}$ can be computed as follows:

$$
t_{p+i-1}=\frac{1}{p-1} \sum_{j=i}^{i+p-2} \bar{t}_{f(j)} \quad(i=1, . ., n-p+1)
$$

where $\overline{t_{k}}$ are the parameters of the points $\mathbf{p}_{k}$ and $f(j)$ is a simple function that returns the index of the point $\mathbf{p}_{k}$ corresponding to a dominant point $\mathbf{d}_{j}$. The knots are computed by averaging the parameters of the dominant points, so the knot vector coincides with the one for B-spline curve interpolation to the dominant points [1].

\subsection{Selection of Dominant Points}

Eventually, the quality of the resulting curve depends on how to select dominant points $\mathbf{d}_{j}(j=0, . ., n)$ among the points $\mathbf{p}_{i}(i=0, . ., m)$. We can devise an algorithm realizing that fewer dominant points are chosen at flat regions but more at complex regions. Its main steps are given as follows:

(1) Set $n_{d}=-1$ where $n_{d}$ denotes the highest index of current dominant points.

(2) Select seed points from the point set, and store them in a list $\mathbf{S}$ in decreasing order of significance. While $\mathbf{S} \neq \varnothing$ AND $n_{d}<n$, pop a point from the list $\mathbf{S}$, make it a new dominant point, and $n_{d} \leftarrow n_{d}+1$.

(3) While $n_{d}<n$, select dominant points $\mathbf{d}_{j}$ adaptively as follows:

(3.1) Find two successive dominant points $\mathbf{d}_{k}=\mathbf{p}_{s}$ and $\mathbf{d}_{k+1}=\mathbf{p}_{e}(|e-s|>1)$ whose corresponding segment incurs the largest deviation.

(3.2) Choose a point $\mathbf{p}_{w}(s<w<e)$ as a new dominant point, and $n_{d} \leftarrow n_{d}+1$.

We herein consider two kinds of seed points: two end points and local curvature maximum points (LCM). We detect LCM points from the curvatures estimated at the given points. Two end points are the most significant. For LCM points, the greater their curvatures are, the more significant they are. 
With the current dominant points, we can generate a current curve by knot placement and least-squares minimization. For two neighboring dominant points $\mathbf{d}_{j}=\mathbf{p}_{s}$ and $\mathbf{d}_{j+1}=\mathbf{p}_{e}$, we can build a segment $\mathbf{S}_{s, e}=\left\{\mathbf{p}_{k} \mid k=s, . ., e\right\}$. Among the segments consisting of at least three points, we find the segment with the largest deviation to the current curve. We then choose a new dominant point from the segment. A simple approach for the choice is to select the median of the points of the segment, but a more promising approach is to select a point $\mathbf{p}_{w}$ that minimizes $\left|\lambda_{s, w}-\lambda_{w, e}\right|$ for $s<w<e$ where $\lambda_{s, e}$ denotes the shape complexity of a segment $\mathbf{S}_{s, e}$ that can be estimated by combining the total curvature and the arc length of the segment.

\section{Experimental Results}

Fig. 1 shows cubic B-spline curve fitting to a point set that consists of 251 points. The points are enclosed in an $84 \times 126$ rectangle. In Fig. 1(d), KTP selects the knots such that each knot span contains almost the same number of parameters. In Fig. 1(e), NKTP shows a similar trend except at the first and the last knot spans. In Figs. 1(c) and 1(f), the proposed approach creates fewer dominant points (curve segments) at flat regions but more at complex regions.

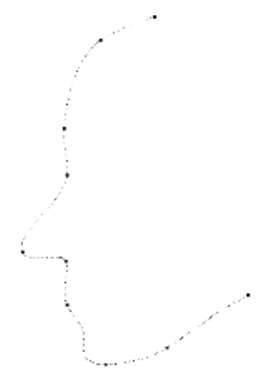

(a)

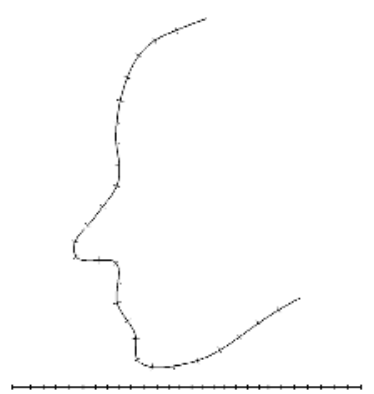

(d)

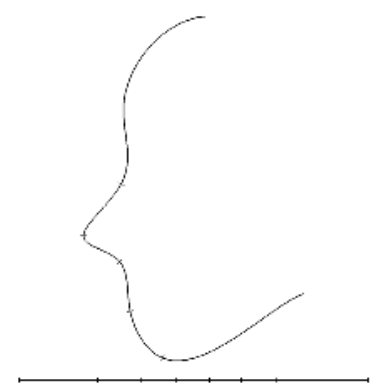

(b)

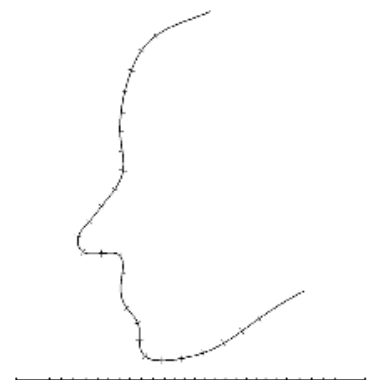

(e)

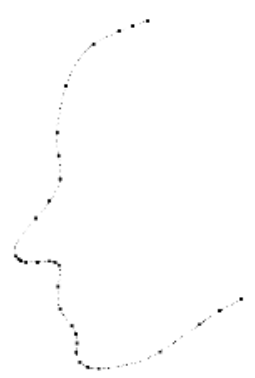

(c)

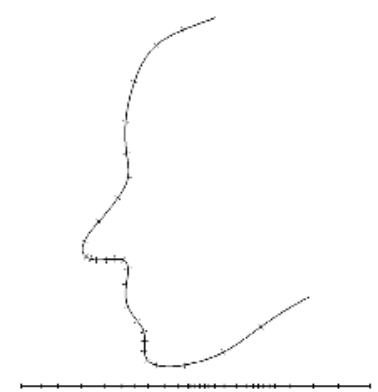

(f)

Fig. 1. B-spline curve fitting: (a) point set with 10 seed points; (b) curve obtained using 10 seed points as dominant points; (c) point set with 31 dominant points; (d) curve obtained by KTP ( $n=31)$; (e) curve obtained by NKTP ( $n=31)$; (f) curve obtained with 31 dominant points 


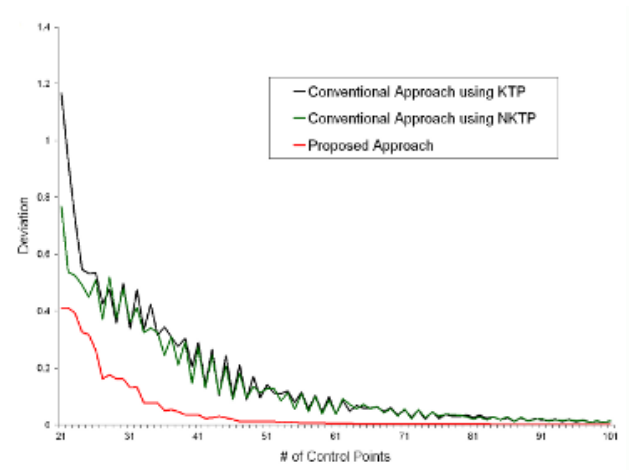

(a)

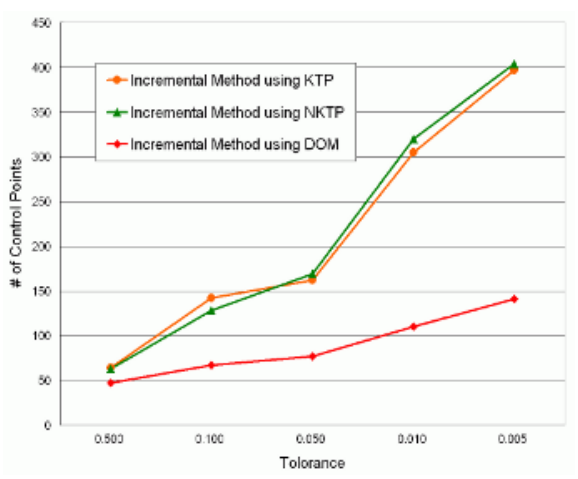

(b)

Fig. 2. Plots for comparison: (a) Deviation plot; (b) Plot of the number of control points

Fig. 2(a) shows the deviation caused by B-spline curve fitting to the point set in Fig. 1(a). The deviation is computed as we increase the number of control points from 16 to 251 . Deviations for more than 101 control points were omitted from the plot since they are not differentiable visually. The deviation caused by the proposed approach is $69 \%$ less than the one caused by the approach using KTP, and the deviation caused by the approach using NKTP is nearly the same as (actually $3 \%$ greater than) the one caused by KTP. Additionally, we adopted three approaches KTP, NKTP, and DOM in the incremental method for the error-bounded curve approximation [3]. DOM denotes the proposed approach. The incremental method starts with few control points and adds control points repeatedly to maintain the error bound $[1,3]$. We compared the three approaches by the number of control points required to approximate the point set within a tolerance. Fig. 2(b) shows the comparison result of the number of required control points. Note that DOM outperforms the others in data reduction.

\section{Concluding Remarks}

Because of the page limit of the paper, we had to shorten the description of the proposed approach to the minimum. The approach can realize the concept of adaptive refinement that fewer curve segments are generated at flat regions but more at complex regions. It can generate a B-spline curve in good quality with less deviation. Adopted in the error-bounded curve approximation, the approach can play an important role in generating B-spline curves with much less control points.

There still remain important issues not covered in this paper: properties of the knot placement using dominant points; non-deterministic algorithms such as genetic algorithms for the optimal selection of dominant points; expansion of the proposed approach to B-spline surface fitting. We expect that all the issues will be handled by the future research. 


\section{References}

1. Piegl, L., Tiller, W.: The NURBS Book. Springer-Verlag, New York (1995)

2. Piegl, L., Tiller, W.: Surface Approximation to Scanned Data. The Visual Computer 16 (2000) 386-395

3. Park, H.: An Error-Bounded Approximate Method for Representing Planar Curves in BSplines. Computer Aided Geometric Design 21 (2004) 479-497 